\title{
ANÁLISIS DE LA OBRA EL MANANTIAL (AYN RAND, 1943). DETECCIÓN DE MODOS DE HACER ARQUITECTURA EN RELACIÓN CON EL TIEMPO
}

Francisco A. GIORDANO (1) / Anna I. LANCELLE (2) / Sergio A. FERNÁNDEZ (3)

giordancisco@gmail.com / annalancelle@yahoo.com.ar / sergio_arquitectura@yahoo.com.ar

1. Becario de pregrado SGCyT 2012-2013; becario pregrado EVC CIN 2014-2015, / 2. Directora de beca, magíster UPC, Prof. adjunta HyCIHyCII, / 3. Codirector de beca, magíster UPC, Prof. responsable Morfología III, JTP Morfología II.

Palabras Clave: Tiempo; arquitectura; historia.

Keywords: time; architecture; history.

\section{RESUMEN}

Se parte del desarrollo de dos proyectos consecutivos ${ }^{1}$, sobre la existencia de distintas concepciones de tiempo que implicarían modos diferenciados de concebir la historia y el hacer arquitectura. Se investigó, en el ámbito arquitectónico, focalizando en el análisis de El Manantial, de AYN RAND.

\begin{abstract}
It is based on the development of two consecutive projects on the existence of different conceptions of time involve different ways of conceiving the history and making architecture. This research, in the field of architecture, was focusing on the analysis of "The Fountainhead", by Ayn Rand.
\end{abstract}




\section{OBJETIVOS GENERALES}

- Construir un cuerpo teórico que permita analizar el texto y el film seleccionados, a partir de los resultados de las investigaciones anteriores, de los antecedentes que el grupo de investigación ha constituido con sus respectivos trabajos y de la bibliografía instrumental.

- Detectar las nociones que expresen modos de hacer arquitectura y sus implicancias filosóficas, según el marco antes mencionado.

- Distinguir estas nociones según a qué concepción de tiempo (sucesión o duración) corresponden los modos de hacer arquitectura puestos en evidencia en los objetos de estudio seleccionados.

- Fundamentar la distinción anterior a partir del cuerpo teórico construido y del análisis de cada uno de los modos de operar implicados en el texto. - Identificar los distintos modos de operar en obras de arquitectura.

\section{INTRODUCCIÓN}

Según los antecedentes, se tendría un modo de hacer historia y arquitectura abstracto, por ideas, al que llamaremos convencionalmente "Historia" o "Sucesión", y un modo de hacer más concreto, casi corporal, al que llamaremos "Duración". Estos dos modos se caracterizan de la siguiente manera:

Sucesión: se entiende aquí a la ciencia histórica que aboga por la certidumbre y las causalidades; es la historia explicativa, progresiva, en la que lo importante son los datos recogidos y su ilación lineal en una secuencia lógica. Memoria del intelecto, cuya expresión más acabada se encuentra en la temporalidad (noción de tiempo convencional). Duración: noción estudiada y desarrollada por HENRI BERGSON, autor referente del que se ha tomado este concepto. Es el tiempo en el cual no existe presente, pasado y futuro, sino un único tiempo percibido corporalmente en el que no hay causas ni consecuencias, sino un acontecimiento único que no puede ser explicado intelectualmente, pues solo ocurre en un presente dilatado. Memoria del cuerpo, cuya expresión más completa se encuentra en la espacialidad (noción de tiempo no convencional).

Dice BERGSON en La percepción del cambio: "Nuestra conciencia nos dice que cuando hablamos de nuestro presente pensamos en cierto intervalo de duración. ¿Qué duración? Es imposible fijarla exactamente; es algo demasiado fluctuante. Mi presente, en este momento, es la frase que pronuncio. Pero es así porque me place limitar a mi frase el campo de mi atención. Esta atención es cosa que puede alargarse y reducirse, como el intervalo entre las dos puntas de un compás. Por el momento las dos puntas se separan justamente lo bastante para ir del comienzo al fin de mi frase; pero si se me antoja alejarlas más, mi presente abrazará, además de mi última frase, aquella que la precedía: habría bastado adoptar otra puntuación" (BERGSON, 1972).

Así, lo que denominamos presente es lo que coincide con el campo de atención actual. Sigue entonces: "Avancemos más: una atención que fuera indefinidamente extensible tendría bajo su mirada, con la frase precedente, todas las frases anteriores de la lección, los acontecimientos que la han precedido y una porción tan grande como se quiera de lo que llamamos nuestro pasado. La distinción que hacemos entre nuestro presente y nuestro pasado es, pues, si no arbitraria, por lo menos relativa a la extensión del campo que puede abarcar nuestra atención a la vida. (...) En una palabra, nuestro presente cae en el pasado cuando cesamos de atribuirle un interés actual" (BERGSON, 1972).

Sobre la base de estas nociones, en investigaciones y trabajos del grupo de investigación se ha 
"...en las creaciones artísticas están implícitos modos de concebir el arte y la arquitectura, lo que implica un cuestionamiento profundo de orden filosófico y ético absolutamente actual, que coinciden en un todo con los modos de comprender el tiempo y el hacer que se corresponde con ellos."

desarrollado una base conceptual teórica resumida en invariantes filosóficas encontradas en autores de la denominada bibliografía instrumental, que forman parte de los proyectos acreditados, como por ejemplo W. BENJAMIN, F. NIETZCHE, H. BERGSON, B. SPINOZA, que permitieron dilucidar el contraste entre estos dos modos de pensar el tiempo y esbozar sus implicancias tanto en la historiografía como en la arquitectura.

En el presente trabajo, se propuso analizar un texto no académico ni perteneciente a la currícula de las asignaturas de historia, sino devenido del ámbito artístico-literario, que además tiene también su contrapartida en la expresión cinematográfica, con el fin de detectar nociones teórico-filosóficas que - pertenecientes específicamente ahora al ámbito de la arquitectura y no a la historiografía (debido a la temática del libro en cuestión) - pudieron relacionarse con los conceptos abordados en la bibliografía instrumental, e incluso aportar otros nuevos, siempre con el fin de comprender modos de hacer arquitectura.

La novela de AYN RAND El Manantial y el film del mismo nombre del director KING VIDOR, que conserva una cuidada fidelidad con el texto original, han sido considerados clásicos por los propios arquitectos y los críticos de arquitectura, quienes no soslayaron la alusión al brillante arquitecto FRANK LLOYD WRIGHT y a sus posicionamientos éticos respecto de la disciplina; pero más allá de la lectura de sus contemporáneos, se sostiene que en estas obras están implícitos dos modos de concebir el arte y la arquitectura que implican un cuestionamiento filosófico y ético absolutamente actual, que coinciden en un todo con los modos de comprender el hacer arquitectónico y sus correspondientes concepciones de tiempo, expuestas más arriba, y que resultan de las indagaciones realizadas hasta el momento en el marco del proyecto acreditado.
El trabajo se realizó, entonces, en el sentido de establecer cuáles eran las nociones teóricofilosóficas contenidas en el texto y en la película que tuvieran que ver con los modos de hacer arquitectura mencionados en el inicio, intentando contrastarlas también con algunos principios obtenidos de la bibliografía instrumental utilizada en los proyectos a fin de evaluar el posicionamiento de la autora y el sentido de la construcción teóricofilosófica propuesta. Finalmente, se in-tentó visualizar las nociones relativas al hacer arquitectura y su comprensión implícita del tiempo en ejemplos de arquitectura clásica, moderna y contemporánea, aludiendo a los referentes universales en la medida en que el estudio así lo fue demandando.

\section{HIPÓTESIS DE TRABAJO}

Se sostuvo que también en las creaciones artísticas —como en la novela El Manantialestán implícitos modos de concebir el arte y la arquitectura, lo que implica un cuestionamiento profundo de orden filosófico y ético absolutamente actual, que coinciden en un todo con los modos de comprender el tiempo (sucesión o duración) y el hacer que se corresponde con ellos.

\section{METODOLOGÍA}

El trabajo se comportó como una investigación de tipo exploratoria en sus diferentes aspectos: -Hermenéutico-Interpretativo: las obras (novela y film) fueron estudiadas y analizadas a la luz del instrumento conceptual obtenido en trabajos anteriores ${ }^{1}$,

1 Se hace alusión aquí al PI N. ${ }^{\circ}$ C003-2009 que enmarca este trabajo y en su propuesta de continuación, el PI C002-2013, sobre la existencia de distintas concepciones de tiempo, que llevaría a modos diferenciados de concebir la historia, el enseñar y el hacer arquitectura:

- un modo abstracto que aboga por la concepción de una realidad fragmentada y que, en relación con la arquitectura, decantaría en un hacer interesado en los resultados formales o lingüísticos.

-un modo corporal, en el que existe un tiempo dilatado y que, en arquitectura, correspondería a un hacer interesado en el proceso. 
esto es, la distinción de dos nociones opuestas de tiempo y de hacer arquitectura.

-Comparativo: las implicancias de uno y otro modo de entender el tiempo y el espacio han surgido del contraste entre el análisis de las obras seleccionadas y textos de índole teórico- filosófica que formaron parte de la bibliografía instrumental.

-Crítico-Evaluativo: a partir de las comparaciones realizadas, se intentó comprender y fundamentar las virtudes y limitaciones de la visión de la autora para llegar a una valoración probada de sus implicancias, especialmente en lo que respecta al proceso de proyecto en arquitectura.

\section{DESARROLLO}

Además de los presupuestos teóricos de los que se vale, explicitados previamente, se exponen aquí las etapas que metodológicamente debieron desarrollarse para llegar a los objetivos propuestos.

\section{Etapa de reconocimiento e investigación}

En primer lugar, se abordó una instancia de investigación referida al contexto histórico y filosófico de la obra en cuestión, en la cual se realizó un estudio analítico y biográfico de la autora y su filosofía.

Por otra parte, se procedió con la lectura del libro El Manantial, de AYN RAND. Esta etapa fue eminentemente de relevamiento de información. Se indagó sobre los antecedentes literarios, biográficos y otras obras, películas y libros de la autora, además de comentarios generales, críticas y opiniones sobre la obra. Se atendió especialmente a críticas referidas a la transposición libro-película, así como a artículos relacionados con la filosofía de la autora.
En una segunda instancia y en paralelo, se investigó además sobre los conceptos y nociones referidos al marco de los proyectos generales de investigación mencionados, los que se refieren básicamente al tiempo y la duración, de los cuales se extrajeron conceptualizaciones concernientes a los diferentes paradigmas contemporáneos: teorías de sistemas, principios biológicos, físicos y filosóficos tales como sinergia, entropía, entelequia, autopoiesis, recursividad, homeostasis, multiplicidad, etc.

\section{Primera etapa de elaboración, general deductiva}

Se realizó un primer abordaje deductivocomparativo-sintético sobre los conceptos y principios pertinentes a los distintos modos de hacer, a un nivel dimensional macro general titulado: el modo de hacer como finalidad en sí versus el modo de hacer como medio para llegar a algo. Esto tiene su antecedente en lo expresado por el filósofo GILLES DELEUZE sobre el acontecimiento: lo que sucede, el verdadero acontecer, se da entre los estados de cosas, no es un ser sino un devenir; en el verdear, en el infinitivo y no el sustantivo (DELEUZE, 1989).

La siguiente dimensión incluye una comparación sobre las distintas actitudes del hacer según la concepción filosófica objetivista de la autora, en la cual se plantea la dicotomía de "el hacer en sí" dentro del eje "razón-individualismo-capitalismo" en oposición a "el hacer para" dentro del eje "misticismo-altruismo-colectivismo".

Finalmente, en una tercera dimensión se analizaron los distintos modos de hacer según los personajes principales de la obra: Howard Roark, el hombre como fin en sí mismo, el hacer en sí mismo, en oposición a Peter Keating, el hombre como medio para los fines de los otros, el hacer para llegar a algo. 


\section{Segunda etapa de elaboración, exhaustiva inductiva}

Se volvió a analizar particularmente la película, pero a un nivel más exhaustivo y a través de un abordaje inductivo.

El mismo constó de las siguientes instancias;

1. Un análisis específico, cronológico, literal y objetivo con algunas interpretaciones al margen.

2. Un cuadro-síntesis comparativo ordenado.

1. Metodología del análisis cronológico.

Descripción de lo que se ve y lo que se escucha.

1.1. Registro de los diálogos más significativos.

1.2. Registro de imágenes sobre las escenas más significativas.

-Interpretación del diálogo y la fotografía.

-Distinción de las dos posiciones filosóficas encontradas.

-Explicación (implicancias con los términos surgidos de la película).

El cuadro-síntesis comparativo constituyó en sí mismo una tercera etapa...

\section{Tercera etapa de elaboración, síntesis analógica}

Cuadro-síntesis comparativo de los dos modos de hacer, extraído del análisis exhaustivo en el cual se distinguen claramente los dos modos, el del hacer en sí mismo y el del hacer por llegar a un resultado.

\section{Cuarta etapa de elaboración. Implicancias filosóficas según otros autores}

En esta etapa se realizó, en una primera instancia, una lectura y distinción en profundidad de un cuerpo-base bibliográfico de tipo filosófico compuesto principalmente por las siguientes obras:

- El hombre mediocre, de José Ingenieros. A partir de este texto se distinguieron dos tipos de hombres: el "hombre-mediocre" y el "hombreideal". "Hay climas morales, horas, momentos, en que toda una raza, un pueblo, una clase, un partido, una secta concibe un ideal y se esfuerza por realizarlo. Y los hay en la evolución de cada hombre, aisladamente considerado.

"Hay también climas, horas y momentos en que los ideales se murmuran apenas o se callan: la realidad ofrece inmediatas satisfacciones a los apetitos y la tentación del hartazgo ahoga todo afán de perfección."

"Cada época tiene ciertos ideales que presienten mejor el porvenir, entrevistos por pocos, seguidos por el pueblo o ahogados por su indiferencia, ora predestinados a orientarlo como polos magnéticos, ora a quedar latentes hasta encontrar la gloria en momento y clima propicio" (INGENIEROS, 2004).

- La rebelión de las masas, de JOSÉ ORTEGA Y GASSET. Aquí se distinguieron dos tipos de hombres: el "hombre-masa" y el "hombre-ejemplar". "Triunfa hoy sobre toda el área continental una forma de homogeneidad que amenaza consumir por completo aquel tesoro. Dondequiera ha surgido el hombre-masa de que este volumen se ocupa, un tipo de hombre hecho de prisa, montado nada más que sobre unas cuantas y pobres abstracciones, y que, por lo mismo, es idéntico de un cabo de Europa a otro. A él se debe el triste aspecto de asfixiante monotonía que va tomando la vida en todo el continente. Este hombre-masa es el hombre previamente vaciado de su propia historia, sin entrañas en el pasado y, por lo mismo, dócil a todas las disciplinas llamadas 'internacionales'" (ORTEGA Y GASSET, 1994). 
- La sociedad del espectáculo, de GUY DEBORD. En esta obra se contrastaron en profundidad todas las implicancias de la noción de "espectáculo". La vida como acontecimiento en oposición a la vida como espectáculo. "Toda la vida de las sociedades donde rigen las condiciones modernas de producción se anuncia como una inmensa acumulación de espectáculos. Todo lo que antes se vivía directamente, se aleja ahora en una representación" (DEBORD, 1974).

En una segunda instancia se sintetizaron las nociones surgidas de aquellos textos y se las comparó con los modos surgidos del objeto de estudio y entre ellas mismas.

Estos autores y sus obras fueron seleccionados en particular debido a la coincidencia en el planteo de base con lo explicitado por AYN RAND en El Manantial. INGENIEROS y ORTEGA Y GASSET han indagado en sus obras cumbres El hombre Mediocre y La rebelión de las masas, respectivamente, sobre esta característica constitutiva del hombre de tender a la masificación y la vulgaridad como consecuencia de la ausencia total de autoexigencia y de anteponer los derechos por sobre las obligaciones.

Más adelante, y en un contexto muy similar al contemporáneo, DEBORD avizora las consecuencias de esta masificación asociada ya al mundo de la mercantilización, lo que denominará "La sociedad del Espectáculo", paradigma del estado de cosas reinante previo al mayo francés y cuyo devenir no ha hecho más que radicalizar y ratificar aquella visión. Entre las primeras obras y esta última, puede situarse el libro de RAND, cuestionado, cuando no ignorado, por evidenciar quizá demasiado descarnadamente el conflicto entre la masificación y la individualidad, que además se desarrolla en el ámbito de la arquitectura.

\section{Quinta etapa de elaboración. Implicancias en ejemplos de arquitectura}

En esta etapa se analizaron en profundidad textos, ensayos y entrevistas de carácter específicamente arquitectónico, a partir de la misma metodología; se procedió mediante analogías y dicotomías, el contraste de los diferentes modos de hacer en la arquitectura universal. En lo contemporáneo se tomó como ejemplificador, por la claridad de las diferencias en sus procesos de diseño, el par MIRALLES (con su arquitectura diversa, corporal, sensorial) y VIAPLANA (con su arquitectura abstracta, premeditada, arquitectura del encargo telefónico); mientras que en la arquitectura clásica se analizó el par BORROMINI (con sus iglesias moldeadas de ladrillo y estuco, que van haciendo espacio escapando a la geometría) y BERNINI (con sus iglesias enfundadas en mármol, en las que la geometría base es evidente).

Para lograr esta síntesis de posicionamientos dentro de lo estrictamente arquitectónico, se utilizó como base textos de JOSEP QUETGLAS (QUETGLAS, 1991) y GIULIO ARGAN (ARGAN, 1984) autores que han desarrollado, en distintos momentos, conceptos ligados concretamente al "arquitecturar", noción que alude al hacer en sí mismo (proceso) por encima del hacer con una finalidad (resultado), el verbo por sobre el sustantivo, los que finalmente se contrastaron y asimilaron a las posiciones evidenciadas en el objeto de estudio y en el cuerpo filosófico analizado. Respecto de ello: "Yo afirmo lo siguiente: la obra de Enric Miralles es imaginario arquitectónico sin filtraciones, en estado puro, es producción y conducción directa del arquitecturar. Por eso sus dibujos, plantas, secciones y escritos siempre vienen después de la imaginación, después de la arquitectura, y pueden ser solo aproximaciones que no preceden sino que siguen al arquitecturar, sin poder coincidir exactamente con él. A su arquitectura le es propia una exactitud que 
"Quien se detiene en el hacer concebirá una arquitectura que es presencia, que produce acontecimientos, mientras que quien hace solo pensando en los resultados representa una arquitectura que determina comportamientos de vida y no permite que las cosas acontezcan."

no es la de la medida (...) Si se tratara de emparejar la arquitectura de Viaplana con alguna pintura de similar raíz, la elección tendría que ir a Salvador Dalí. Dalí no es un pintor —como Wagner, decía Nietzsche, no es un músico-. En Dalí, la pintura siempre es un a posteriori, es el documento que certifica que una ocurrencia brillante ha tenido lugar, antes, en otra parte - por tanto, fuera de la pintura, previa al pintar- El cuadro aparece como vehículo subsidiario de la ocurrencia. Es un monumento conmemorativo de la fantasía que se ha tenido. Igual con Viaplana" (QUETGLAS, 1991).

\section{RESULTADOS}

\section{En relación con los objetivos}

Respecto de los objetivos planteados, se llegaron a concretar de manera satisfactoria construyendo un cuerpo teórico que permitió analizar el texto y el film seleccionados, a partir de los resultados de las investigaciones anteriores, de los antecedentes que los miembros del grupo de investigación han constituido con sus respectivos trabajos y de la bibliografía instrumental. Se detectaron luego las nociones que expresan modos de hacer arquitectura y sus implicancias filosóficas, según el marco antes mencionado, distinguiendo así estas nociones según a qué concepción de tiempo (sucesión o duración) corresponden los modos de hacer arquitectura puestos en evidencia en los objetos de estudio seleccionados. Existiría, según ello, un modo que destaca lo procesual, en el que la concepción del tiempo es la de duración, mientras habría también un modo de proyectar finalístico en la que la concepción del tiempo es lineal. Se llegó entonces a detectar las implicancias de estos modos según a qué concepción de tiempo (sucesión o duración) correspondieran. Quien se detiene en el hacer concebirá una arquitectura que es presencia, que produce acontecimientos, mientras que quien hace solo pensando en los resultados representa una arquitectura que determina comportamientos de vida y no permite que las cosas acontezcan.

Se valoró según estas implicancias qué modo de hacer arquitectura se corresponde con una práctica de la profesión pertinente e integral. Ello permitió aproximarse a los principios o características que debería tener el modo de hacer arquitectura que se corresponde con la concepción de tiempo más adecuada según la valoración anterior.

\section{En relación con la hipótesis}

Considerando la hipótesis de partida: "Se sostiene que también en las creaciones artísticas como en la novela El manantial están implícitos modos de concebir el arte y la arquitectura que implican un cuestionamiento profundo de orden filosófico y ético absolutamente actual, que coinciden en un todo con los modos de comprender el tiempo (sucesión o duración) y el hacer que se corresponde con ellos", se ha podido constatar que, desde el estudio de los fundamentos filosóficos surgidos de los textos interpretados, el análisis exhaustivo del film y el de las obras literarias y artísticas colaterales a este, existen en El manantial de manera implícita y explícita distintos modos opuestos de concebir tanto el arte como la arquitectura, regidos esencialmente por principios que concretan dos visiones antagónicas del mundo.

\section{En relación con la etapa de reconocimiento e investigación y a la primera etapa de elaboración general deductiva}

Respecto de esta primera etapa puede decirse que significó el encuentro con el texto de base del film y con lo que suscitó tanto la publicación del libro como el estreno de la película. Se comprendió así por qué fue, y aún sigue siendo, 
una obra resistida, en tanto se la lee solo como una apología del individualismo, sin reparar en las profundas implicancias éticas y estéticas que el conflicto representado plantea: la obtención de resultados a toda costa en contraposición con el disfrute en el hacer.

La lectura en paralelo de algunos capítulos de Lógica del Sentido, obra del filósofo contemporáneo GILLES DELEUZE, echó luz sobre tal distinción, al analizar especialmente nociones como devenir, o acontecimiento. Esto permitió en esta etapa partir de la ruptura del prejuicio instalado sobre El Manantial para orientar la investigación hacia aquellas nociones cercanas al filósofo. Es así que se concluyó en la absoluta coincidencia entre el accionar del protagonista de Rand y lo que DELEUZE Ilama "el infinitivo", el verbo siempre actual, sin finalidad.

En relación con la segunda etapa de elaboración, exhaustiva inductiva, y a la tercera etapa de elaboración, síntesis analógica

El análisis minucioso del libro y el registro pormenorizado de los fotogramas y los diálogos del film han permitido distinguir claramente dos modos de hacer:

-un modo de hacer preocupado por el proceso, por la experiencia;

-un modo de hacer con una finalidad, preocupado por los resultados.

En relación con la cuarta etapa de elaboración. Implicancias filosóficas según otros autores

Se verificó también que estas nociones se relacionan y son coincidentes con lo desarrollado en otras fuentes bibliográficas de distintos ámbitos, lo que ha contribuido a explicitar que tanto en la obra literaria de base como en el film analizados existen dos modos de hacer contrapuestos que devienen también de dos modos de ser de hombres también opuestos.

A partir de la lectura de los textos que forman parte de la bibliografía, se arribó a las nociones y características más significativas que presentan cada uno de ellos en el desarrollo de sus distintas temáticas. A través de la comparación de las nociones de allí surgidas, se encontraron similitudes y diferencias entre las distintas posturas de los autores analizados y entre estos y la autora de la obra de base, AYND RAND.

Desde la primera parte de esta investigación, y en las primeras distinciones surgidas del cuerpo directo, empezaron a vislumbrarse ciertos términos que podrían interpretarse apresuradamente como discordantes, como: el idealismo, el individualismo, el egoísmo, entre otros, que fueron despejados claramente en los primeros textos de la bibliografía seleccionada (El hombre mediocre y La rebelión de las masas). Otros, como la cuestión de estilo, relacionado con el eclecticismo y la modernidad exhibidos en la obra, fueron contrastados con los textos que involucraron, necesariamente, una mirada más competente y precisa que excedió a lo propiamente arquitectónico.

En una primera instancia, se distinguieron dos tipos de hombres, dos caracteres-temperamentos, en los cuales el carácter del protagonista de la obra Howard Roark ${ }^{2}$ se corresponde con el modo de ser idealista de INGENIEROS y el modo ejemplar, noble de ORTEGA, mientras que Peter Keating ${ }^{3}$ es su opuesto. Progresivamente, esta contraposición se vuelve menos evidente en los sucesivos textos, y a mayor complejidad pueden aflorar ciertas contradicciones que solo pueden ser despejadas

2 Arquitecto, protagonista de la obra El manantial, encarna los valores referidos al tiempo propio o tiempo proceso.

3 Arquitecto, antagonista en la obra, corporiza los valores opuestos al protagonista, tanto en lo personal como en su arquitectura, el tiempo sucesivo, con miras a un resultado. 

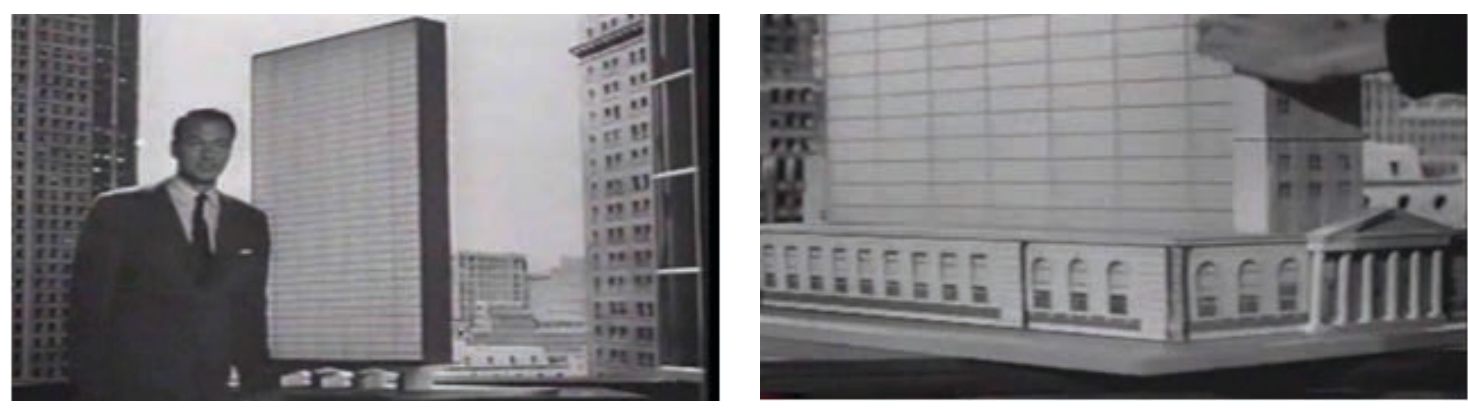

Maqueta del edificio propuesto por Howard. La misma propuesta con lenguaje clásico adosado por la Junta del Security Bank

por la lectura y compresión de las obras en su totalidad. Por ende, las posibilidades de relación y conexiones entre ellas son muchas, por lo que se prosiguió con la diferenciación para agrupar las ideas, que se entendió son afines entre sí.

Estos dos tipos o modos de ser de hombres han podido distinguirse en:

-el hombre idealista en general, el hombre superior de INGENIEROS (INGENIEROS, 2004); el hombre ejemplar de ORTEGA (ORTEGA Y GASSET, 1994);

-el hombre no idealista en general, el hombre mediocre de INGENIEROS, el hombre masa de ORTEGA.

Haciendo un paralelo con la obra de base y la película, solo a Howard Roark le corresponde el modo de ser idealista, ejemplar, no espectacular. Su estilo tiene que ver con un modo de hacer, no con una herencia de lenguajes y formas. Su técnica es la variación, la multiplicidad, la superposición (se da por entendido). Es el "hacer en sí", el proceso, el diálogo. Por lo tanto, hace una arquitectura ideal, ejemplar, excelente, no espectacular, viva, íntegra.

En la misma, a Peter Keating y los "otros" — como contraposición a Howard Roark - les corresponde el modo de ser mediocre, masa, vulgar, espectacular. Su estilo es la herencia de un lenguaje y formas pre-existentes. Su técnica es la mímesis; su idea, la imposición. Es el "hacer para", la búsqueda del resultado. Por lo tanto, producen una arquitectura mediocre, masa, vulgar, espectacular, muerta, fragmentaria.

En relación con la quinta etapa de elaboración. Implicancias en ejemplos de arquitectura

Según lo anterior, solo a Howard Roark (equivalente en el análisis posterior a Miralles o a Borromini) le corresponde el modo de ser y hacer corporal, el arquitecturar como infinitivo, el tiempo-proceso o tiempo-duración, el hacer en sí (lo "idealista", "ejemplar", en palabras de ORTEGA Y GASSET, lo "no espectacular" en el texto de GUY DEBORD); mientras que a Peter Keating (equivalente en los últimos ejemplos analizados a Viaplana o a Bernini) le corresponde el modo de ser y hacer abstracto, solo intelectual, la arquitectura como resultado, como sustantivo, el hacer para un fin (lo "mediocre" en términos de JOSÉ INGENIEROS, lo "espectacular" para GUY DEBORD).

En este sentido, en cuanto a los resultados obtenidos, es decir, los modos de ser de las arquitecturas resultantes, se ha podido distinguir:

- Arquitectura no espectacular, según el texto de GUY DEBORD (DEBORD, 1974), de la imaginación (QUETGLAS, 1991).

- Arquitectura espectacular, según el texto de GUY DEBORD (DEBORD, 1974), de la ilusión (QUETGLAS, 1991).

En relación con las características principales de cada uno de esto modos en arquitectura y de sus principales artífices en el Barroco, dice ARGAN: "Bernini parte de la idea de que únicamente el diseño realiza su ideal teórico y de que la traducción práctica sólo puede ser una aproximación; por tanto, trata de que esta aproximación sea lo más cercana posible al ideal y considera que será más valiosa a través del empleo de los materiales considerados nobles. Borromini, en cambio, quiere poner en evidencia el valor de espiritualidad del proceso diseño-construcción en su continuidad, y no necesita un material que se imponga por una atribución tradicional de nobleza, sino que sea extremadamente dúctil, sensible a su modelación lineal" (ARGAN, 1984).

En el mismo libro, la comparación se aplicó también al Renacimiento y su clasicismo en oposición al Barroco y su anticlasicismo, valores que se habían hecho evidentes en los dos arquitectos, Bernini y Borromini, respectivamente. A partir de esto, pudieron diferenciarse entonces: 

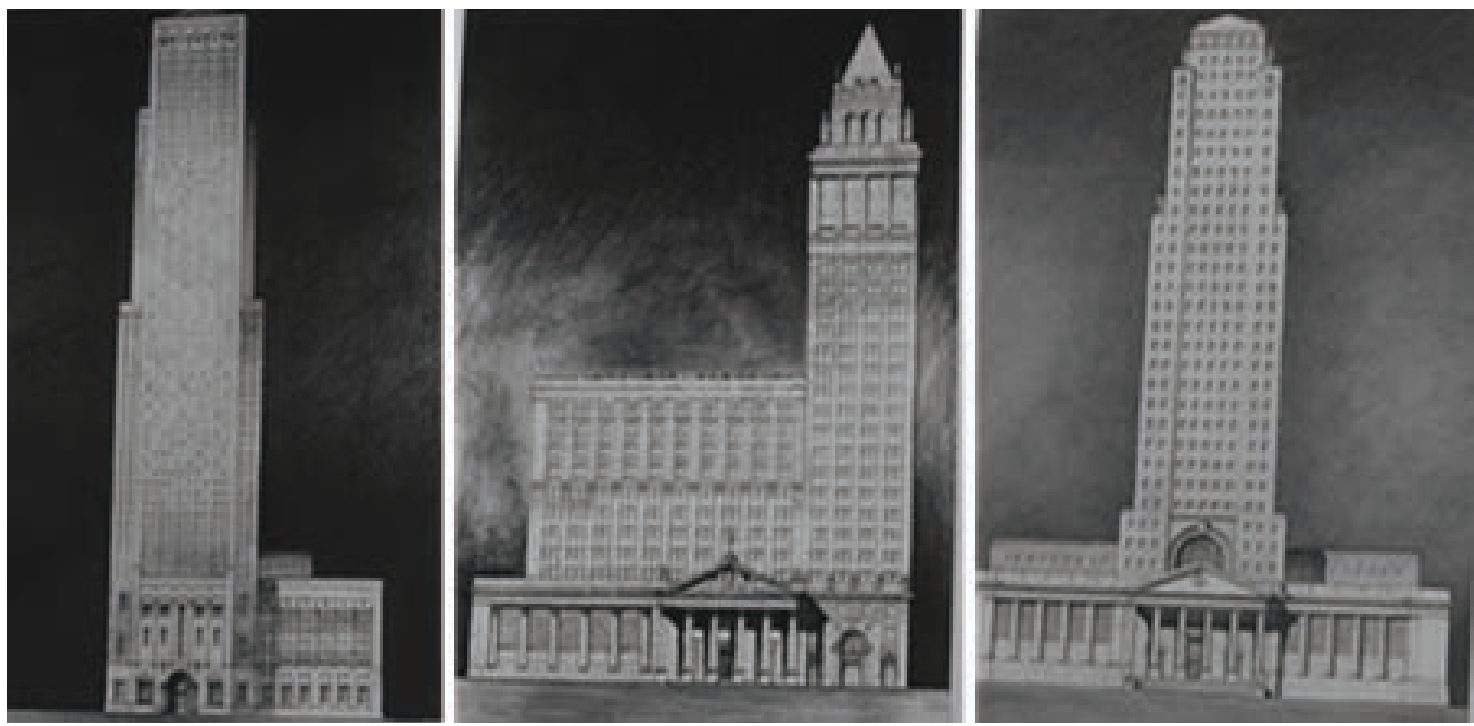

Diferentes fachadas sobre espacios modernos que re-presentan el Clasicismo imperante en pasajes del filme

- Arquitectura de la determinación del espacio: que se va haciendo en relación con su materialización, que no representa una idea previa, que hace delirar la técnica.

- Arquitectura de la representación del espacio: que es independiente de su materialización, que representa una idea previa, que se somete o somete a la técnica.

No obstante, es necesario insistir que este proceder a modo de distinción no implica la intención de una suerte de reduccionismo, sino más bien una manera de poder congregar y generalizar ciertas cuestiones que por su complejidad sería imposible afrontar de otro modo.

\section{FINALMENTE...}

Así, habiendo hecho el análisis en ejemplos de la arquitectura universal tanto clásica como contemporánea y relacionando estos resultados con los dos modos encarnados por los personajes de la película, puede decirse que Howard Roark es el tipo de hombre-arquitecto que coincide en su carácter temperamento con Borromini y Miralles. Por tanto, la arquitectura de Howard Roark posee unas características que coinciden con la arquitectura de Borromini y Miralles. También puede afirmarse que Peter Keating y los "otros" son los tipos de hombres-arquitectos que coinciden en sus caracteres temperamentos con Bernini y Viaplana. Por tanto, la arquitectura de Peter Keating y los "otros" posee unas características que coinciden con la arquitectura de Bernini y Viaplana.

Los sucesivos análisis y búsquedas de ejemplos, guiados por los resultados de los trabajos del grupo de investigación insertos en los proyectos mencionados antes, han sido arduos y extensos, lo que solo permitió llegar a efectuar las comprobaciones de los conceptos surgidos de la obra de base (libro y película) en ejemplos paradigmáticos de la arquitectura universal tanto antigua como contemporánea.

\section{REFERENCIAS BIBLIOGRÁFICAS}

ARGAN, Giulio Carlo (1984) El concepto del espacio arquitectónico desde el barroco a nuestros días. Ed. Nueva Visión, Buenos Aires.

BENJAMIN, Walter (1989) Tesis de Filosofía de la Historia. En. Discursos interrumpidos. Ed. Taurus, Buenos Aires.

BERGSON, Henri (1912) La evolución creadora. Renacimiento Editorial, Madrid.

BERGSON, Henri (1972) El pensamiento y lo moviente. Ed. la Pléyade Buenos Aires.

BERGSON, Henri (1979) Introducción a la metafísica. Ed. Siglo Veinte, Buenos Aires.

DÉBORD, Guy (1974) La sociedad del espectáculo. Ed. De La Flor, Buenos Aires.

DELEUZE, Gilles (1989) Lógica del sentido. Ed. Paidós, Barcelona.

INGENIEROS, José (2004) El hombre mediocre. Ed. Agebe, Buenos Aires NIETZSCHE, Friedrich. (1959) Consideraciones Intempestivas. Ed. Aguilar, Buenos Aires.

ORTEGA Y GASSET, José (1994). La rebelión de las masas. En Obras Completas. Tomo IV. Ed. Alianza, Madrid.

QUETGLAS, Josep (1991) "No te hagas ilusiones". En: El Croquis 49-50. En construcción. Enric Miralles / Carme Pinós 1988-1991.

RAND, Ayn (2007) El manantial (versión castellana). Ed. Grito Sagrado, Buenos Aires.

SPINOZA, Baruch (2000) Ética demostrada según el orden geométrico. Ed. Trotta, Madrid.

Fuente imágenes: película "El Manantial", del director King Vidor, de 1949 